\title{
EFECTO DEL FENÓMENO “EL NIÑO 1997-98” EN EL DESEMBARQUE DE LOS RECURSOS PESQUEROS ARTESANALES DE PUERTO SALAVERRY (TRUJILLO)
}

\author{
EFFECT OF "EL NIÑO 1997-98" PHENOMENON IN LANDING \\ OF ARTISANAL FISHERY RESOURCES OF SALAVERRY (TRUJILLO)
}

Alvaro Tresierra-Aguilar, Zoila Culquichicón y Bilmia Veneros-Urbina*

\section{ABSTRACT}

The results of direct consultations about the landing volumes of artisanal fishery is analyzed in Salaverry Port, before, during and after "El Niño 1997-98" phenomenon.

The landings during the first phase of "El Niño" were about of $1000 \mathrm{tm}$ in 1996 and it increased to $17000 \mathrm{tm}$ in 1997. The presence of tropical water species with "El Niño" event intensification the first semester of 1998 was increased in fishery and more than $50 \%$ of landings corresponded to dolphinfish. Later on, the use landing volumes of traditional species returned with normalization of environmental conditions. The socioeconomic impact is also analyzed.

La pesquería artesanal en el Perú es una actividad económica y social de gran importancia, brinda trabajo a más de 20000 pescadores y surte a la población peruana con más de 280000 tm de pescado fresco (Zapata, 1987). Según Walsh (1981), la zona norte del Perú es la que tiene los mayores puntos de desembarque y la mayor variedad de especies pelágicas y demersales. El Puerto de Salaverry es uno de los más importantes del Departamento de La Libertad, en cuanto al volumen de desembarques.

En el presente trabajo se analiza el resultado de consultas directas sobre los volúmenes de desembarque de la pesquería artesanal que opera en el Puerto Salaverry, antes, durante y después del fenómeno " $\mathrm{El}$

* Departamento Académico de Pesquería - Univ. Nac. de La Libertad. Aptdo. 952. Trujillo, Perú
Niño 1997-98". Los datos de temperatura fueron obtenidos de la Dirección Regional de Pesquería.

En 1996, los desembarques en el Puerto de Salaverry alcanzaron cerca de $1000 \mathrm{tm}, \mathrm{y}$ los mayores porcentajes correspondieron a Sciaena deliciosa (loma), Isacia conceptionis (cabinza), Mugil cephalus (lisa), Prionace glauca (tiburón azul) y Sphyrna zygaena (tiburón martillo); y entre los crustáceos y moluscos, a Platyxanthus orbignyi (cangrejo violáceo) y Octopus sp. (pulpo) (Tabs. I y 2).

En 1997, en la primera fase de "El Niño", los volúmenes de desembarque llegaron a $17000 \mathrm{tm}$. La composición de la pesca se caracterizó por mayores porcentajes de Sardinops sagax sagax (sardina), Scomber japonicus (caballa), Sarda chiliensis chiliensis (bonito) y Coryphaena hippurus (dorado); y 
Tabla 1. Peces presentes en los desembarques en el Puerto Salaverry.

\begin{tabular}{|c|c|c|c|c|c|}
\hline $\begin{array}{l}\text { Especie } \\
\text { Nombre común }\end{array}$ & $\begin{array}{l}\text { Año } \\
\text { Nombre especifico }\end{array}$ & 1996 & 1997 & $1998 \mathrm{~A}$ & $1998 B$ \\
\hline Angelote & Squatina armata & + & + & & + \\
\hline Anguila & Ophichthus pacifici & + & & & \\
\hline Aguja & Strongylura exilis & & + & & \\
\hline Anchoveta/peladilla & Engraulis ringens & & + & & \\
\hline A tún aleta amarilla & Thunnus albacares & & + & + & \\
\hline Bagre & Galeichthys peruvianus & + & + & & + \\
\hline Bonito/chauchilla & Sarda chiliensis chiliens is & + & + & + & + \\
\hline Borracho & Scartichthys gigas & + & & & \\
\hline Barbudo & Polydactylus approximans & & + & + & \\
\hline B arrilete & Katsuwonus pelamis & & + & + & \\
\hline Barrilete negro & Auxis rochei & & + & + & \\
\hline Cachema & Cynoscion analis & + & + & + & + \\
\hline Chita & Anisotremus scapularis & + & + & + & + \\
\hline Chiri & & + & + & & \\
\hline Corvina & Cilus gilberti & + & & + & \\
\hline Cherlo & Acanthistius plotus & + & + & + & \\
\hline Cazón & Carcharhinus sp. & + & + & & \\
\hline Caballa & Scomber japonicus & & + & & + \\
\hline Cabinza & Isacia conceptionis & + & + & & + \\
\hline Cabrilla & Paralabrax sp. & + & + & & \\
\hline Caballito de $\mathrm{mar}$ & Hipocampus inges & & & & + \\
\hline Cojin oba & Seriolella violacea & + & + & & \\
\hline Congrios & Genypterus sp. & + & + & & \\
\hline Corvina cherela & Cynoscion phoxocephalus & + & + & & \\
\hline Corvina dorada & Micropogonias altipinn is & + & + & & \\
\hline Cabezón & Caulolatilus cabezon & + & & & \\
\hline Dotado & Coryphaena hippurus & + & + & + & \\
\hline Guitarra & Rhinobatos planiceps & + & + & + & + \\
\hline Gallinaza & Ophioscion obscurus & + & + & + & \\
\hline Jurel & Trachurus picturatus murphyi & + & + & & \\
\hline Lenguado & Paralichthys adspersus & + & + & + & + \\
\hline Lis a & Mugil cephalus & + & + & + & + \\
\hline Lorna & Sciaena deliciosa & + & + & & + \\
\hline Mojarrilla & Stellifer minor & + & + & + & \\
\hline Mojarra & Gerres cinereus & + & & & \\
\hline Misho & Menticirhus ophicephalus & + & + & + & \\
\hline Machete & Ethmidium chilcae & + & + & + & \\
\hline Merluza & Merluccius gayiperuanus & + & + & + & \\
\hline Mero & Ephinephelus sp. & + & + & & \\
\hline Pámpano & Trachinotus paitens is & + & + & & + \\
\hline Pejegalio & Callorhinchus callorynchus & + & & & \\
\hline Pejerrey & Odonthestes regia regia & + & + & & \\
\hline Pezcinta & Trichiurus nitens & & & & + \\
\hline Pez loro & Oplegnathus insignis & + & + & & \\
\hline Pintadilla & Cheilodactylus variegatus & + & + & + & \\
\hline Raya & Myliobatis sp. & + & + & + & + \\
\hline Robalo/robatillo & Sciaena starski & + & + & + & + \\
\hline Sardina & Sardinops sagax sagax & + & + & & \\
\hline Suco & Paralonchurus peruanus & + & + & + & \\
\hline Sierrá & Scomberomorus maculatus & & + & + & + \\
\hline Viña & Menticirrus sp. & + & + & + & \\
\hline Tiburón azul & Prionace glauca & + & + & + & + \\
\hline Tiburón diamante & Isurus oxyrinchus & + & + & & \\
\hline Tiburón martillo & Sphyrina zygaena & + & + & & \\
\hline Trambollo & Labrisom us philippi & & + & + & \\
\hline Tollo & Mustelus sp. & + & + & & \\
\hline
\end{tabular}


Tabla 2. Crustáceos, moluscos y otras especies presentes en los desembarques en el Puerto Salaverry.

\begin{tabular}{|c|c|c|c|c|c|}
\hline $\begin{array}{l}\text { Especie } \\
\text { Nombre comün }\end{array}$ & $\begin{array}{l}\text { Año } \\
\text { Nombre especifico }\end{array}$ & 1996 & 1997 & 1998A & 1998B \\
\hline Cangrejo violáceo & Platyxanthus orbignyi & + & + & & \\
\hline Langosta & Panilurus gracilis & + & + & & + \\
\hline Jaiba & Toxotes sp. & & + & & \\
\hline Langostino & Penaeus sp. & & & + & \\
\hline Almeja & Gari solida & & + & & \\
\hline Choro & Aulacomya ater & + & & & \\
\hline Calamar & Loligo sp. & + & + & & + \\
\hline Caracol negro & Thais chocolata & & + & & \\
\hline Pota & Dosidicus gigas & + & + & & \\
\hline Pulpo & Octopus sp. & + & + & & \\
\hline Chancho marino & & + & + & & \\
\hline Lobo marino & & + & & & \\
\hline Tortuga verde & & + & + & & \\
\hline
\end{tabular}

una disminución sustancial en la mayoría de especies tradicionales (lorna y cabinza). La lisa y el tiburón azul mantuvieron su volumen. Entre los invertebrados aparecieron Thais chocolata (caracol negro), Loligo sp. (calamar) y Gari solida (almeja) (Tab. 2).

En el primer semestre de 1998 (1998A) las mayores temperaturas alcanzadas por el evento
"El Niño" (Fig. 1), tuvo una clara manifestación en la presencia de especies de aguas tropicales en la pesca, como el Scomberomorus maculatus (pez sierra), Katsuwonus pelamis (barrilete), dorado, atunes y tiburones (Tab. 1). Más del $50 \%$ de los desembarques correspondió al dorado; y el langostino Penaeus sp. alcanzó

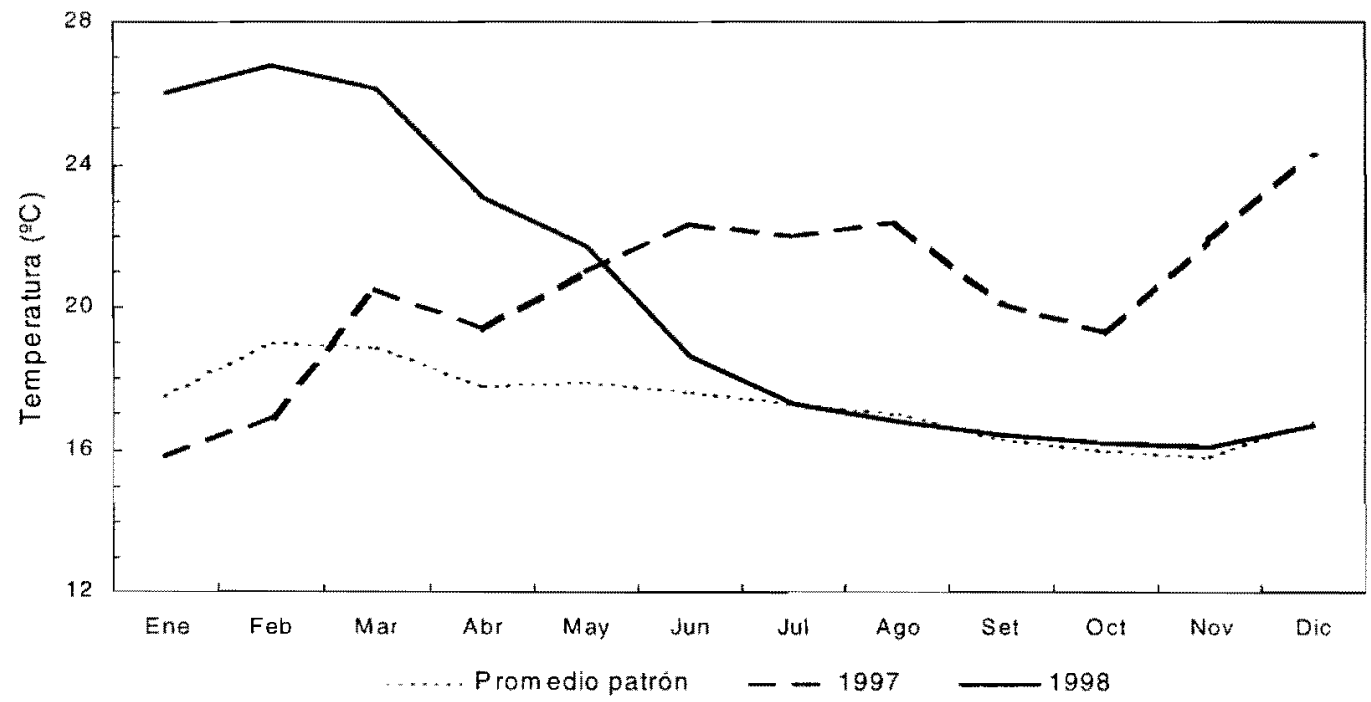

Figura 1. Temperatura superficial del mar en el Puerto de Salaverry durante los años 1997, 1998 y el promedio patrón obtenido para el período 1930-97. 
desembarques inusuales.

En el segundo semestre de 1998 (1998B) las condiciones ambientales tendieron a la normalidad y las especies tradicionales, como Cynoscion analis (cachema), caballa, cabinza y lorna, volvieron a sus volúmenes de desembarque habituales (Tab. 1).

Impactos similares fueron registrados en anteriores eventos "E1 Niño". Así, Schweigger (1964) registró en "El Niño 1941" igual resultado "invasión de especies tropicales" como el atún de aleta amarilla (Thunnus albacares) y barrilete ( $K$. pelamis), que se capturó incluso en la Caleta de Pucusana. En 1965, se observó otra vez la invasión de atún de aleta amarilla, barrilete, dorado y otras especies tropicales. Durante "El Niño 1982-83", las condiciones fueron diferentes al de los eventos anteriores, el sistema pelágico estaba compuesto por un mayor número de especies sometidas a diferente grado de explotación, como durante el evento de 1997-98. Los resultados del presente trabajo coinciden con el incremento del número de especies durante "El Niño 1982-83", regi strado por Valdivia y Arntz (1985).

En general, durante "El Niño 1997-98" la mayoría de especies tradicionales, como la cabrilla (Paralabrax sp.), cachema (Cynoscion analis), lenguado (Paralichthys adspersus), lorna (Sciaena deliciosa), caballa (Scomber japonicus), fueron reemplazadas por especies tropicales de gran tamaño, como atunes, barriletes, dorados y tiburones. Estas nuevas especies fueron aprovechadas por los pescadores artesanales foráneos que tenían la posibilidad de cambiar la red cortina por el espinel, en desmedro de los pescadores locales quienes vieron afectada su economía. Asimismo, la presencia y captura de langostinos favoreció a muchos pescadores y empresarios foráneos, que congelaron el producto para la exportación.

\section{LITERATURA CITADA}

Schweigger, E. 1964. El Litoral Perthano. $2^{\text {a }} \mathrm{ed}$. Universidad Nacional Federico Villarreal, Lima, 414 pp.

Walsh. 1981. Pesca artesanal y recursos costcros. Mnst. Mar Peru-Callao (79):2-23.

Valdivia. E. y W.E. Antz. 1985. Cambios en los recursos costeros y su incidencia en la pesqueria artesanal durante "El Niño" 1982-83. Ln: W.E. Amtz; A. Landa y J. Tarazona (eds.). "El Niño". su impacto en la fauna marina. Bol. Inst. Mar Peri-Callao Vol. Extraor: 143-152.

Zapata. E. 1987. Descripción y situación de las pesquerias artesanales en el Peru. Dirección General de Extracción. Ministerio de Pesqueria. 22 pp. 